\title{
Changes in Cardiac Output and Peripheral Flows on Pentobarbital Anesthesia in the Rat
}

\author{
Yasushi KawaUe and Juro IrIUChIJIMA \\ Department of Physiology, School of Medicine, University of \\ Hiroshima, Hiroshima, Hiroshima, 734 Japan
}

\begin{abstract}
In rats chronically implanted with an electromagnetic flow probe around the ascending aorta, terminal aorta, or superior mesenteric artery as well as arterial and venous indwelling catheters, changes in cardiac output, hindquarter flow, splanchnic flow, and arterial pressure on pentobarbital anesthesia were observed. On intravenous injection of pentobarbital sodium at $30 \mathrm{mg} / \mathrm{kg}$, arterial pressure dropped acutely from an average value of about $105 \mathrm{mmHg}$ to a minimum of about $75 \mathrm{mmHg}$ in about $5 \mathrm{~min}$ and then gradually recovered to an average level of about $90 \mathrm{mmHg}$ in $30 \mathrm{~min}$. Cardiac index gradually decreased about $30 \%$ on the average in $30 \mathrm{~min}$. Hindquarter flow decreased about $25 \%$. Superior mesenteric flow first increased about $40 \%$ within 5 min and then returned almost to the premedication level in $30 \mathrm{~min}$. In adrenalectomized rats there was no decrease of hindquarter flow on pentobarbital anesthesia. The increase in superior mesenteric flow immediately after pentobarbital injection remained almost unchanged after adrenalectomy or splanchnicectomy. It is concluded that an appreciable portion of the decrease in cardiac output on pentobarbital anesthesia is induced by inhibition of tonic adrenomedullary secretion which has a dilating effect on muscle blood vessels through stimulation of $\beta$-receptors. The marked increase in splanchnic flow immediately after pentobarbital injection, which is responsible for the concomitant drop in arterial pressure, is considered to be induced by a direct inhibitory effect of the anesthetic on splanchnic blood vessels.
\end{abstract}

Key Words: anesthesia on circulation, cardiac output, muscle flow, splanchnic flow, arterial pressure.

Although the rat is widely used in medical research, information on the influence of anesthetics on this animal's hemodynamics is relatively meager and confined to observations on arterial pressure and at best on cardiac output (MizoGAMI et al., 1969; Coleman, 1974; SMith and Hutchins, 1980). This constitutes

Received for publication September 30, 1983

川上恭司, 入内島十郎 
an obstacle in interpreting experimental results obtained under anesthesia in this animal.

In a study using an electromagnetic flowmeter of cardiac output and its peripheral distribution in the rat, we previously observed a great discrepancy between the results of an acute experiment under pentobarbital anesthesia and those of a chronic experiment in the conscious state (KAWAUE et al., 1982; IRIUCHIJIMA et al., 1982). The acute experiment was performed in laparotomized rats, while the flow measurement in the chronic experiment was carried out with rats in their usual habitat. It is almost impossible to appraise the effect of the anesthetic by comparing the results of these two experiments.

In the present study, therefore, pentobarbital was injected into rats in their habitual cages, and blood flow and pressure were recorded continuously with chronically implanted electromagnetic flow probes. The effect of pentobarbital on circulation was studied by observing continuous changes in flow and pressure from the conscious state before anesthesia to the state of surgical anesthesia. It was found that an inhibitory effect of pentobarbital on tonic adrenomedullary secretion as well as its direct inhibitory effect on splanchnic blood vessels constitute a major part of the influence of this anesthetic on the circulatory system.

\section{METHODS}

Implantation of electromagnetic flow probe. Male Wistar rats at 10-12 weeks of age were anesthetized with thiamylal sodium $(50 \mathrm{mg} / \mathrm{kg}$, i.p.) for chronic implantation of electromagnetic flow probes and arterial and venous catheterization.

Cardiac output was measured by means of a Biotronex probe with an internal diameter of $2.5 \mathrm{~mm}$. The anesthetized rat was fixed in the supine position. A transverse skin incision was made on the thorax bilaterally along the third rib. The third sternocostal cartilage was cut bilaterally close to the sternum. A part of the sternum was removed for about $5 \mathrm{~mm}$ by transverse sections of the sternum at the level of the second and third intercostal spaces. The bilateral thymi were separated and the ascending aorta was reached through the mediastinum without opening the pleura. The aorta was isolated from the surrounding tissues and the head of the probe was attached to it.

For measurement of flow in the terminal aorta, the rat was placed right side down. A longitudinal skin incision was made from the left iliac crista craniad for about $3 \mathrm{~cm}$. The thoracolumbar fascia was cut and the terminal portion of the abdominal aorta was reached retroperitoneally without opening the abdominal cavity. The aorta was separated from the adjacent vena cava down to the iliac bifurcation. A Nihon Kohden flow probe with an internal diameter of $2 \mathrm{~mm}$ was placed immediately craniad to the bifurcation.

The superior mesenteric artery was reached with the rat placed right side 
down, through a longitudinal skin incision of about $3 \mathrm{~cm}$, caudad from the left costolumboabdominal triangle also retroperitoneally, after cutting the internal and external abdominal oblique fasciae. The artery was separated from the adjacent tissues to place a Nihon Kohden flow probe with an internal diameter of $1 \mathrm{~mm}$.

Only one flow probe was implanted in each rat. From the head of each flow probe, the lead wire to the plug was passed under the skin and led to the dorsal neck to exteriorize the plug through a skin incision. The portion of the wire adjacent to the plug was sutured to the neck muscles so that the plug was fixed on the skin in the dorsal neck after suturing the skin wound.

Catheterization. The catheter for intravenous injection was prepared by fusing a polyethylene tube having an internal diameter of $0.35 \mathrm{~mm}$ and an external diameter of $1.05 \mathrm{~mm}$ (PE20, 7405, SP19) to a polyethylene tube of $0.28 \mathrm{~mm}$ (i.d.) and $0.61 \mathrm{~mm}$ (e.d.) (PE10, 7400, SP10) in hot air. The thinner part was left inside the external jugular vein and the thicker part was led to the dorsal neck and exteriorized. Except in the rat with a probe implanted at the terminal aorta, a catheter for recording arterial pressure, which was prepared with the same polyethylene tubes as those for the venous catheter, was inserted from the femoral artery to the terminal aorta and the other end was also led to the dorsal neck to exteriorize.

Adrenalectomy. In some rats, simultaneously with probe implantation, the bilateral adrenals, both medulla and cortex, were removed retroperitoneally after opening the bilateral costolumboabdominal triangles. Thereafter, twice a day every $12 \mathrm{hr}, 0.2 \mathrm{mg}$ of an adrenocortical hormone dexamethasone sulfate was intravenously injected.

Splanchnicectomy. In some of the other rats, splanchnicectomy was performed prior to probe implantation. Rats were anesthetized with thiamylal sodium $(50 \mathrm{mg} / \mathrm{kg}$, i.p.). Laparotomy was performed by an incision along the linea alba. The bilateral splanchnic nerves were cut cephalad to the celiac ganglion. The adrenal branches of the nerve were also severed. In the rats thus splanchnicectomized, probe implantation and catherization were performed as in other rats after about 1 week with recovery of the general condition.

Measurement. After probe implantation the rat was kept separately in a white polyethylene cage of $35 \times 30 \times 17 \mathrm{~cm}$ in size containing wood chips. Water and pellets were given ad libitum.

Flow and pressure measurements were commenced when more than 2 days had passed after implantation and the rat had resumed taking ample water and pellets.

One example of the blood flow pattern recorded at the ascending aorta is presented in Fig. 1A. The diastolic interval was used to approximate zero flow. The flow signal was integrated by a CR circuit with a time constant of $1 \mathrm{sec}$ so that continuous change of cardiac output could be followed as the change of de- 


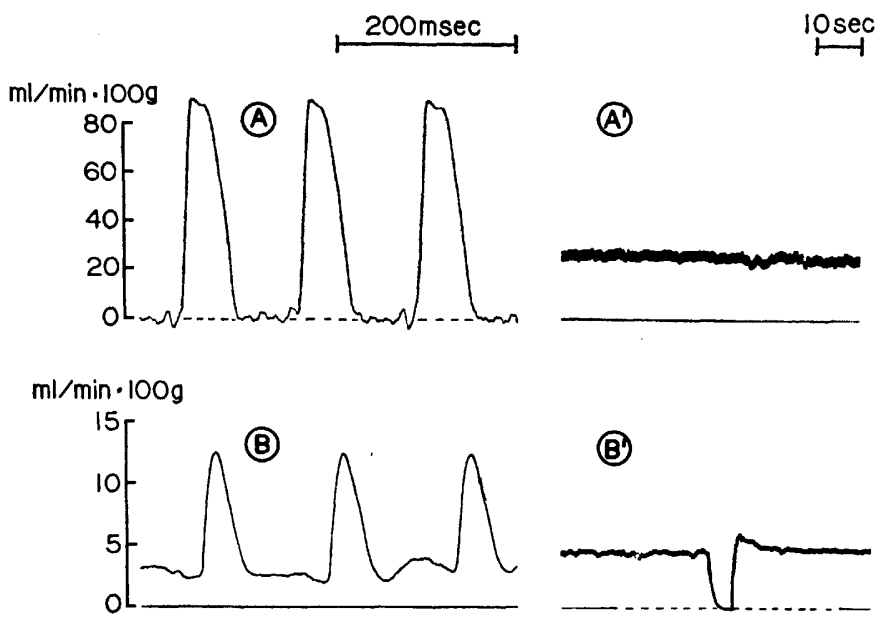

Fig. 1. Flow in the ascending aorta (A) and in the superior mesenteric artery (B) recorded with chronically implanted electromagnetic flow probes in rats. $A^{\prime}$ and $B^{\prime}$ are respective mean flows obtained by integration with a time constant of $1 \mathrm{sec}$. At the center in $\mathbf{B}^{\prime}$, the zero flow level was defined by digital compression of the artery.

flection from the zero flow level as shown in Fig. 1A'.

Figure 1B shows one example of the flow patterns recorded at the superior mesenteric artery. The signal here was also integrated with a time constant of $1 \mathrm{sec}$ to obtain the mean flow (Fig. 1B'). The point of zero flow was defined by stopping the flow momentarily by digital compression applied on the skin (Fig. $1 \mathrm{~B}^{\prime}$, center). The flow in the terminal aorta was recorded similarly.

Each flow probe was calibrated before implantation by passing known amounts of saline through an excised aorta or artery.

All flows were normalized to $100 \mathrm{~g}$ of body weight for comparison.

Injection of pentobarbital. A solution of Nembutal sodium, containing $50 \mathrm{mg}$ of barbituric acid derivative of pentobarbital sodium per $\mathrm{ml}$, propylene glycol at $40 \%$, and alcohol at $10 \%$, was injected at a dose of $0.6 \mathrm{ml} / \mathrm{kg}$ body weight (30 $\mathrm{mg} / \mathrm{kg}$ of pentobarbital sodium) in about $5 \mathrm{sec}$ through the venous catheter. Then the catheter was flushed with about $0.05 \mathrm{ml}$ of $0.9 \%$ saline. Thereafter, changes of blood flow and pressure were continuously recorded for $30 \mathrm{~min}$. The effects of alcohol and propylene glycol on the circulatory system were judged negligible because the quantities were small $(0.12 \mathrm{~g} / \mathrm{kg}$ for alcohol and $0.48 \mathrm{~g} / \mathrm{kg}$ for propylene glycol). After intravenous injection of the anesthetic, an operable state was attained almost immediately and the rats did not recover consciousness for at least $1 \mathrm{hr}$. 


\section{RESULTS}

\section{Changes of arterial pressure and cardiac output on anesthesia}

Figure 2A shows one example of recording of arterial pressure and cardiac output when the rat was anesthetized with intravenous injection of $30 \mathrm{mg} / \mathrm{kg}$ of pentobarbital sodium. Figure 3 summarizes the results of the same experiment carried out in 9 rats.

Arterial pressure rapidly decreased after injection and reached a minimum level in about $5 \mathrm{~min}$. Thereafter, it recovered gradually and stabilized at a level slightly below the premedication control level. On the other hand, cardiac output gradually decreased and reached a level $30 \%$ below the control in $30 \mathrm{~min}$. Total peripheral resistance, calculated by dividing arterial pressure by cardiac output, decreased significantly 5 min after injection and then gradually increased to reach a level higher than the premedication control level. Heart rate increased slightly on anesthesia.

Rats were in a state of typical surgical anesthesia 30 min after the intravenous injection of pentobarbital. This is the stage when acute experimentation under anesthesia is usually performed. On the average, $30 \mathrm{~min}$ after pentobarbital
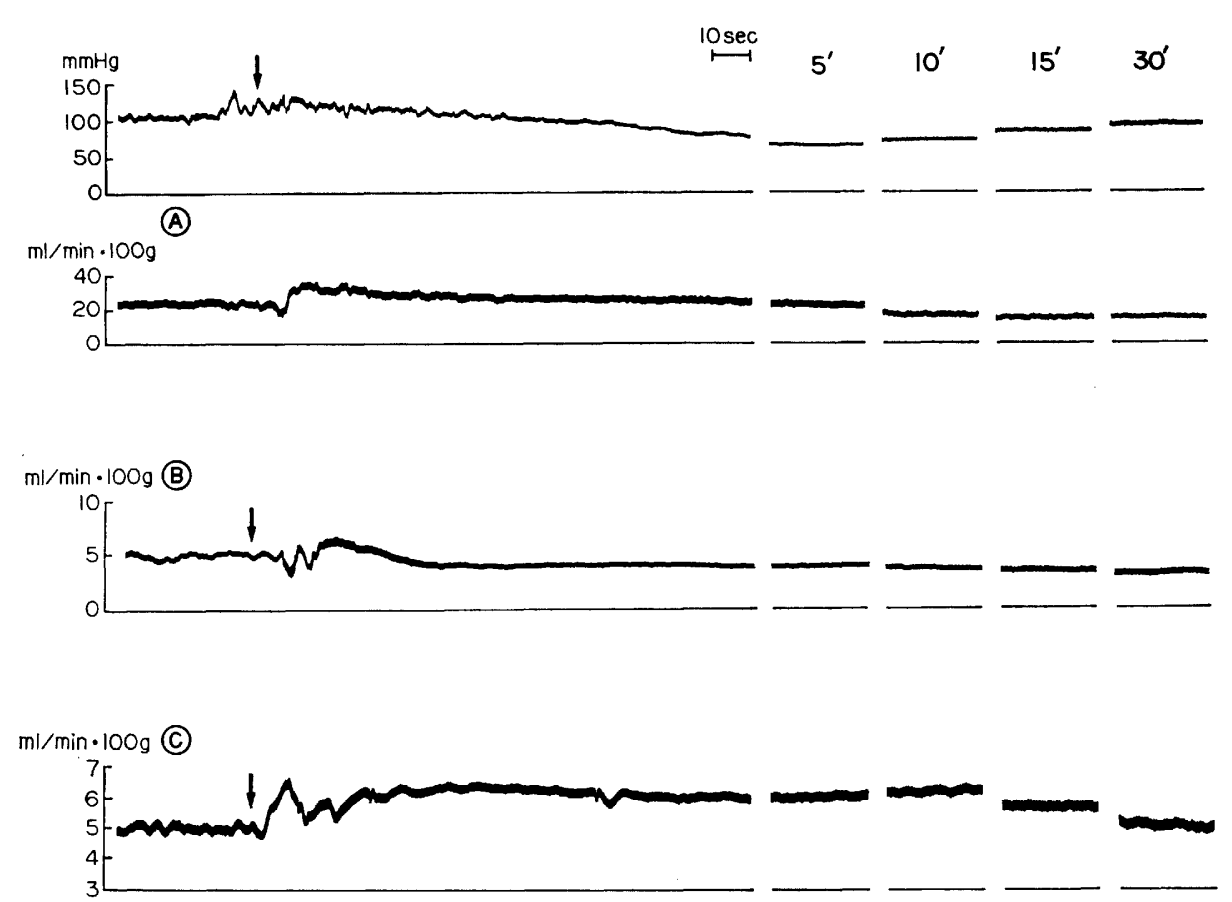

Fig. 2. Changes in (A) arterial pressure and cardiac output (flow in the ascending aorta), (B) flow in the terminal aorta (hindquarter flow), and (C) superior mesenteric flow on intravenous injection of pentobarbital, $30 \mathrm{mg} / \mathrm{kg}$, at the arrows. $\mathrm{A}, \mathrm{B}$, and $\mathrm{C}$ are from different rats.

Vol. 34, No. 2, 1984 

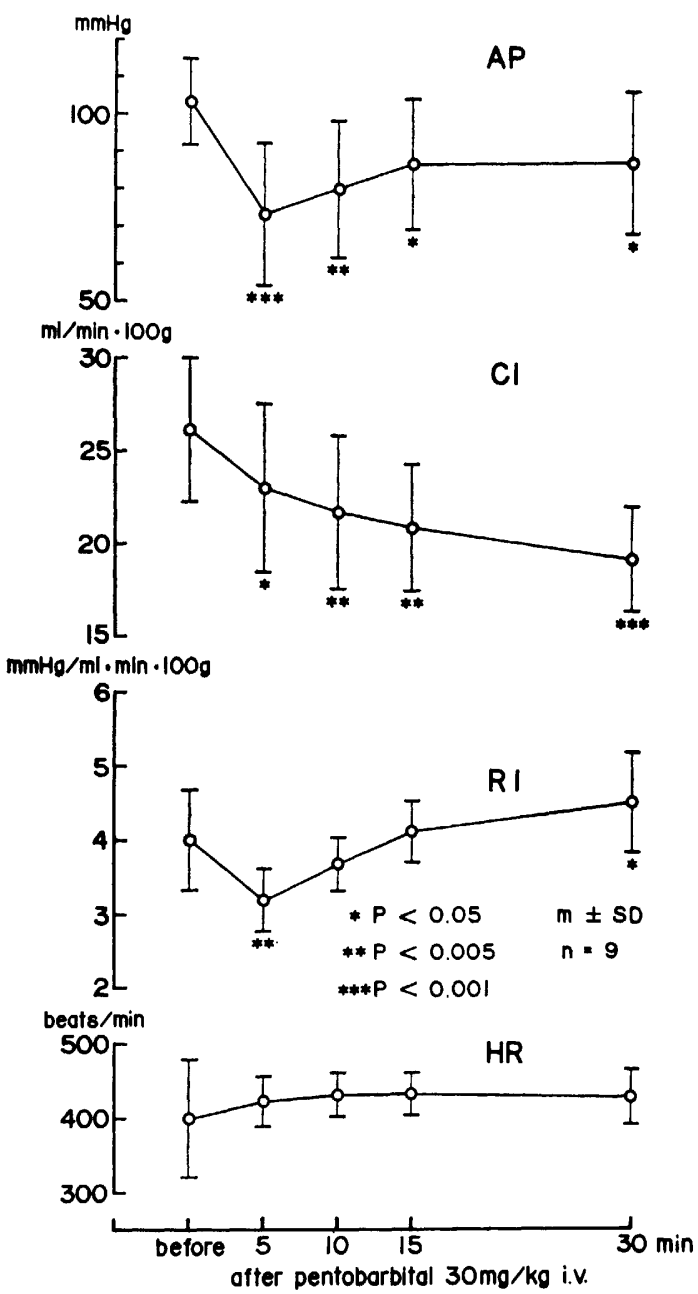

Fig. 3. Successive changes of arterial pressure (AP), cardiac index (CI), total peripheral resistance index (RI), and heart rate (HR) by pentobarbital anesthesia. Asterisks indicate level of significant change from premedication value.

injection, arterial pressure was about $15 \%$ less, cardiac output about $30 \%$ less, and total peripheral resistance about $15 \%$ more than the corresponding control values.

Flow in terminal aorta (hindquarter flow)

The flow recorded at the terminal aorta is referred to as "hindquarter flow." A majority of it consists of flow in skeletal muscles in the bilateral lower extremities.

Figure $2 \mathrm{~B}$ shows one example of the change in hindquarter flow on injection 


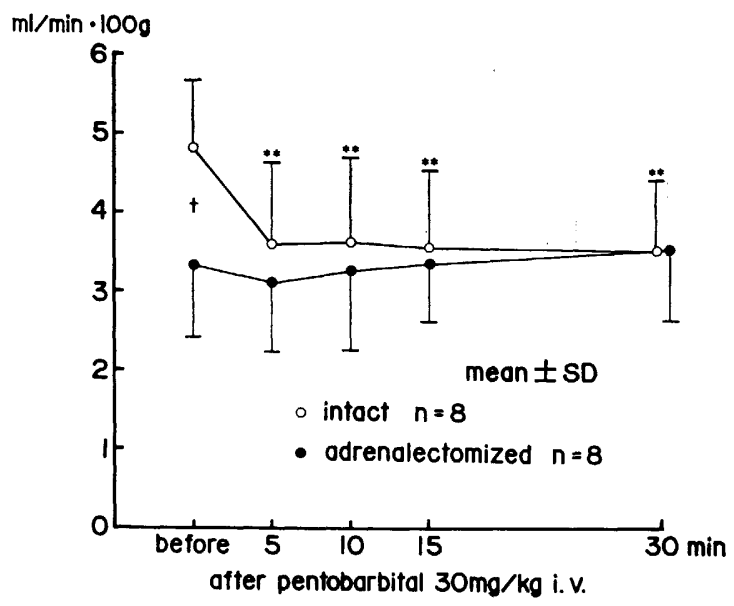

Fig. 4. Changes in flow in the terminal aorta by pentobarbital anesthesia.

** Significant decrease from the value before anesthesia at $p<0.005$, ${ }^{\dagger}$ significant difference between intact and adrenalectomized rats at $p<0.005$.

of pentobarbital. In Fig. 4 (open circles) is plotted the time course of the means \pm S.D. of this parameter from 8 rats during the first 30 min after injection. Hindquarter flow decreased about $25 \%$ on anesthesia.

The filled circles in Fig. 4 represent the results of the same experiment carried out in adrenalectomized rats. Before anesthesia at rest hindquarter flow was significantly lower in adrenalectomized rats than in rats with intact adrenals. In adrenalectomized rats, unlike in rats with adrenals intact, hindquarter flow did not change appreciably on pentobarbital anesthesia.

In conscious rats with intact adrenals, $\beta$-adrenoceptor blockade with propranolol $(1 \mathrm{mg} / \mathrm{kg}$, i.p.) decreased the resting level of hindquarter flow by about $20 \%$ (IrIUCHIJIma et al., 1982). After $\beta$-blockade, anesthesia with pentobarbital induced almost no change in hindquarter flow (experiment in 2 rats). When a rat was first anesthetized with pentobarbital and then administered propranolol, the latter medication did not decrease the hindquarter flow any further.

In a rat after combined adrenalectomy and severance of the bilateral lumbar sympathetic chains, the effect of pentobarbital anesthesia on hindquarter flow was similar to that in adrenalectomized rats.

\section{Superior mesenteric flow}

This flow is considered to be a representative splanchnic flow. Figure 2C presents one example of the change of superior mesenteric flow on pentobarbital anesthesia. Figure 5, open circles, shows the summary data from 8 rats.

Immediately after pentobarbital injection, superior mesenteric flow increased markedly for a while, then decreased gradually and almost reached the premedica- 


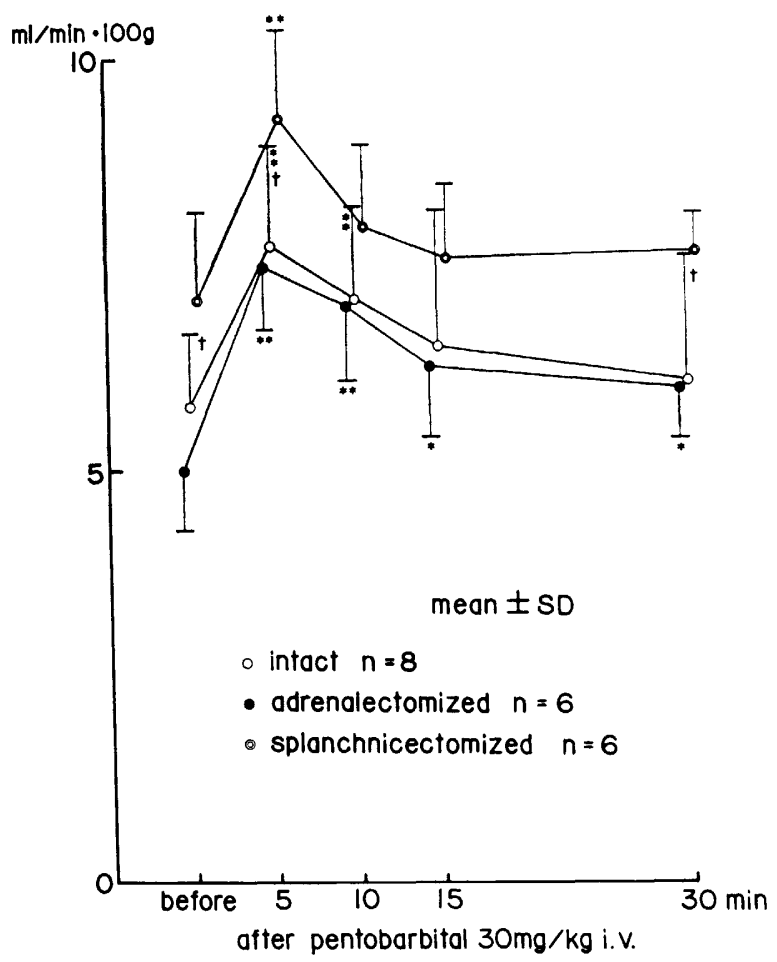

Fig. 5. Changes in superior mesenteric flow by pentobarbital anesthesia.

* Significant change from the value before anesthesia at $p<0.05, * *$ significant change from the value before anesthesia at $p<0.005$, ${ }^{\dagger}$ significant difference between the groups above and below the mark at $p<0.05$.

tion control level in $30 \mathrm{~min}$.

The filled circles in Fig. 5 represent the results of the same experiment performed in adrenalectomized rats. The increase in superior mesenteric flow immediately after pentobarbital injection occurred as in rats with intact adrenals. The following decline of the flow was also similar, but the flow still remained at a level significantly $(p<0.05)$ higher than the premedication control level $30 \mathrm{~min}$ after pentobarbital injection.

The double circles in Fig. 5 represent the changes in superior mesenteric flow on pentobarbital anesthesia in splanchnicectomized rats. Anesthesia also induced a transient increase in flow in these rats. However, the flow level always remained higher than in rats without splanchnicectomy.

\section{DISCUSSION}

Changes in arterial pressure, cardiac output, and total peripheral resistance on pentobarbital anesthesia. That pentobarbital anesthesia decreases blood pressure 
Table 1. Contributions from changes in cardiac output $\left(C_{Q}\right)$ and total peripheral resistance $\left(C_{\mathrm{R}}\right)$ to change in blood pressure in pentobarbital anesthesia calculated from mean values in Fig. 3.

\begin{tabular}{ccc}
\hline & $5 \mathrm{~min}$ & $30 \mathrm{~min}$ \\
\hline$C_{\mathrm{Q}}$ & $42 \%$ & $180 \%$ \\
$C_{\mathrm{R}}$ & $58 \%$ & $-80 \%$ \\
\hline
\end{tabular}

and cardiac output and increases total peripheral resistance as confirmed in the present study has been reported by CoLEMAN (1974) and SMITH and Hutchins (1980).

IRIUCHIJIMA $(1969,1972)$ devised a quantitative indexation of the part played by the change in cardiac output and that by the change in total peripheral resistance in a given change of arterial pressure. Actually the indices, $C_{Q}$ and $C_{\mathrm{R}}$, are quotients of the relative changes of cardiac output and total peripheral resistance to the relative change of arterial pressure. Table 1 shows the indices calculated for the change in arterial pressure on pentobarbital anesthesia from the mean values plotted in Fig. 3. The marked fall of arterial pressure 5 min after pentobarbital injection was contributed to almost equally by the decrease in cardiac output and that in total peripheral resistance. On the other hand, the lowering of arterial pressure $30 \mathrm{~min}$ after injection was entirely due to the decrease in cardiac output. Then the total peripheral resistance had conversely increased to offset the decrease of arterial pressure, as previously reported by other authors (COLEMAN, 1974; SMITH and Hutchins, 1980).

Hindquarter flow. As shown in Fig. 4, the hindquarter flow recorded at the terminal aorta was reduced markedly on pentobarbital anesthesia. This phenomenon was absent in adrenalectomized rats.

We have previously shown that hindquarter flow is markedly increased when the rat is transposed from its habitual cage to a new cage. The major part of this flow increase is ascribable to dilatation of muscle blood vessels due to the $\beta$ effect of adrenaline released from the adrenal medulla (IRIUCHIJIMA et al., 1982). In adrenalectomized rats the increase in hindquarter flow in the response is much smaller. Besides, in adrenalectomized rats, the resting level of hindquarter flow is significantly lower than that in rats with adrenals intact. From this observation we have concluded that, in the rat, blood vessels in skeletal muscles in such parts as the lower extremities are maintained in a dilated state by tonic release of adrenaline even when the rat is apparently at rest. On the other hand, WALKER et al. (1959) has reported that anesthesia inhibits the resting secretion of the adrenal medulla. From these facts, the decrease in hindquarter flow on pentobarbital anesthesia observed in the present study may be ascribable to the inhibition of the resting secretion of the adrenal medulla by the anesthetic and the abolishment of $\beta$ adrenergic vasodilatation in skeletal muscle in the hindquarter area. A considerable portion of the decrease in cardiac output on anesthesia may also be ascribable 
to the decrease in flow in skeletal muscle in the whole body by this mechanism.

Secretion of vasopressin in pressor amounts is also inhibited by pentobarbital anesthesia (IRIUCHIJIMA, 1983). It is interesting that two major regulatory hormones for circulation, adrenomedullary adrenaline and pituitary vasopressin, are both inhibited by pentobarbital.

Superior mesenteric flow. Although superior mesenteric flow $30 \mathrm{~min}$ after pentobarbital injection was almost the same as that before anesthesia, the flow increased markedly soon after injection of the anesthetic, especially after about $5 \mathrm{~min}$. Because this increase was similarly observed after adrenalectomy or splanchnicectomy, it is believed due to the direct inhibitory effect of pentobarbital (Altura and Altura, 1975) on smooth muscle of resistance vessels in the splanchnic area.

Changes in peripheral resistance. The role played by the superior mesenteric resistance in the change in total peripheral resistance on anesthesia was appraised using two indices, $\alpha$ and $\beta$ (IRIUChiJima et al., 1969; IrIUChIJIMA, 1972), The larger the $\alpha$, the greater is the contribution of the peripheral resistance of the particular area to the change in total peripheral resistance, an $\alpha$ of unity indicating that the contribution is on the average. The $\beta$ indicates what percentage of the change of the total peripheral conductance, inverse of total peripheral resistance, is contributed by the particular area. The mean values of pressure and flow were used in the calculation of the indices. The indices were also calculated for the hindquarter resistance. Since arterial pressure was not actually recorded simultaneously with hindquarter flow, the mean value of arterial pressure recorded with superior mesenteric flow was applied for calculation of the indices for hindquarter peripheral resistance.

Indices $\alpha$ and $\beta$ thus calculated are presented in Table 2. Five min after pentobarbital injection, the $\alpha$ index for the superior mesenteric vascular bed was 2 . This indicates that, in the marked decrease in total peripheral resistance at this point, the decrease in resistance in this bed was as intense as twice the average. The corresponding value of 0.26 for hindquarter flow indicates that the decrease in resistance or the vasodilatation in this area was slight. The $\beta$ index for the superior mesenteric area was $58 \%$ and that for the hindquarter area was $4 \%$. This indicates that about half of the increase in total peripheral conductance took place in the superior mesenteric area and that the contribution from the hindquarter

Table 2. Contributions from changes in local peripheral resistance to change in total peripheral resistance in pentobarbital anesthesia.

\begin{tabular}{clcc}
\hline & & $5 \mathrm{~min}$ & $30 \mathrm{~min}$ \\
\hline$\alpha$ & Superior mesenteric & 2.0 & -0.79 \\
& Hindquarter & 0.26 & 1.2 \\
$\beta$ & Superior mesenteric & $58 \%$ & $-22 \%$ \\
& Hindquarter & $4.0 \%$ & $22 \%$ \\
\hline
\end{tabular}


Table 3. Changes in hindquarter peripheral resistance in pentobarbital anesthesia.

\begin{tabular}{lccc}
\hline & $\begin{array}{c}\text { Hindquarter } \\
\text { peripheral } \\
\text { resistance } \\
\text { before } \\
\text { anesthesia }\end{array}$ & $\begin{array}{c}\text { Hindquarter } \\
\text { peripheral } \\
\text { resistance } \\
30 \text { min after } \\
\text { pentobarbital } \\
\text { injection }\end{array}$ & $\%$ change \\
\hline Rats with adrenals intact & 21.5 & 25.1 & +16.7 \\
Adrenalectomized rats & 31.5 & 27.0 & -14.0 \\
\hline
\end{tabular}

Peripheral resistance in $\mathrm{mmHg} /(\mathrm{ml} / \mathrm{min} / 100 \mathrm{~g}$ b.w.).

area was immaterial.

Thirty minutes after pentobarbital injection, the $\alpha$ and $\beta$ indices for the superior mesenteric artery were both negative. This was due to the decrease in superior mesenteric resistance in the face of the increase in total peripheral resistance. The increase in resistance was above average in the hindquarter area. The decrease in conductance in this area was equal in absolute magnitude to the increase in the superior mesenteric area. Since the total peripheral resistance then increased by about $15 \%$, resistance must have been increased in areas other than the hindquarter and superior mesenteric areas.

Table 3 shows the values of hindquarter peripheral resistance before and 30 min after pentobarbital injection, calculated from the mean values of hindquarter flow in Fig. 4 and those of arterial pressure simultaneously recorded with superior mesenteric flow. After adrenalectomy, hindquarter resistance was decreased by pentobarbital anesthesia. This indicates that, in the hindquarter area as in the superior mesenteric area, pentobarbital has a direct inhibitory effect on resistance vessel smooth muscle.

\section{REFERENCES}

Altura, B. T. and Altura, B. M. (1975) Pentobarbital and contraction of vascular smooth muscle. Am. J. Physiol., 229: 1635-1640.

Coleman, T. G. (1974) Cardiac output by dye dilution in the conscious rat. J. Appl. Physiol., 37: 425-455.

IrIUChIJIMA, J. (1969) The constituents of arterial pressure change. Experientia, 25: 713-714. IRIUCHIJIMA, J. (1972) Cardiovascular Physiology, Igaku Shoin, Tokyo, Chap. 3.

IRIUCHIJMA, J. (1983) Conditions for secretion of vasopressin in pressor amounts in waterreplete rats. Jpn. J. Physiol., 33: 887-894.

IriuchiJima, J., KaWAue, Y., and TeRanishi, Y. (1982) Blood flow redistribution in the transposition response of the rat. Jpn. J. Physiol., 32: 807-816.

IrIUChIJIMA, J., KoIKe, H., and MATSUDA, K. (1969) Indices for the role played by regional flow resistance in blood pressure reflex. Pflügers Arch., 307: 22-28.

Kawaue, Y., Teranishi, Y., and Iriuchijima, J. (1982) Blood flow distribution in anesthetized normal and spontaneously hypertensive rats. Jpn. J. Physiol., 32: 299-301.

Mizogami, S., Shibayama, F., Kikuchi, H., and Sokabe, H. (1969) Effects of anesthesias on blood pressure in hypertensive rats. Nihon Taishitsu Igaku Zasshi, 32: 59-63 (in Japanese). 
SMith, T. L. and Hutchins, P. M. (1980) Anesthetic effects on hemodynamics of spontaneously hypertensive and Wistar-Kyoto rats. Am. J. Physiol., 238: H539-H544.

Walker, W. F., Zileli, M. S., Reutter, F. W., Shoemaker, W. C., and Moore, F. D. (1959) Factors influencing the 'resting' secretion of the adrenal medulla. Am. J. Physiol., 197: 765-772. 